\title{
I. Über eine bei der Trennung von Mineralien mittelst schwerer Lösungen zu beachtende Gefahr.
}

\author{
Von \\ W. F. Hillebrand in Washington. \\ Übersetzt von W. Brendler.
}

$\mathrm{DaB}$ die eine oder andere der für die Trennung von Mineralien verwendeten schweren Flüssigkeiten und Lösungen auf einige wenige Mineralien chemisch einwirkt, ist bereits seit langem bekannt. So greift die als $\bowtie$ Sonstadt'sche» oder >Thoulet'sche Lösung " bekannte Lösung von Kaliumquecksilberjodid Metalle und wahrscheinlich auch gewisse Oxyde und Sulfide an. Die ,Rohrbach'sche Lösung " von Baryumquecksilberjodid hat wahrscheinlich ähnliche Wirkung. Die »Kl ein'sche Lösung « von Cadmiumborowolframat wirkt sowohl auf Carbonate als auch auf Metalle ein. Andere schwere Lösungen besitzen zweifellos auch ihre specifische Wirkung.

So weit bekannt war, haben die bisher beobachteten Wirkungen zweifellos zur vollständigen Auflösung oder wenigstens tiefgreifenden Zersetzung des angegriffenen Minerals geführt; eine derartige Einwirkung bringt aber, da sie leicht wahrnehmbar ist, keine ernste Gefahr mit sich. Ich habe nunmehr jedoch über einen Fall zu berichten, in welchem der Angriff einen chemischen Austausch zwischen der schweren Lösung und dem Mineral hervorruft, ohne daß derselbe an letzterem irgend eine mit bloßem Auge sichtbare äußere Veränderung zur Folge hätte.

Vor etwa zwei Jahren wurde mir von der Foote Mineral Company in Philadelphia ein kanariengelbes, krystallisiertes Mineral zur Untersuchung übersandt, das aus dem Paradox Valley, Montrose Co., Colorado, stammte und für Carnotit gehalten wurde, der in der Hauptsache ein wasserhaltiges Kaliumuranvanadat darstellt.

Es ergab sich, daß das Mineral in seiner Zusammensetzung dem Carnotit sehr ähnlich war, nur mit dem Unterschiede, daß das Kalium durch Calcium 
ersetzt war. Später wurde der Versuch gemacht, etwas davon mittelst Kaliumquecksilberjodidlösung zu reinigen; die Analyse ergab dann aber dafür die Zusammensetzung des Carnotits. Um die vermutete Ursache zu prüfen, wurde ein anderes, weniger reines Handstück in zwei Teile geteilt und das eine derselben im Originalzustande analysiert, während das andere erst untersucht wurde, nachdem es 18 Stunden lang der Einwirkung des Quecksilbersalzes unterworfen war. Es steilte sich heraus, dal dieser Teil die Hauptmenge seines Calciumgehaltes verloren und dafür die genau äquivalente Menge an Kalium aufgenommen hatte. Nachstehende Nebeneinanderstellung zeigte die Zusammensetzung der beiden Teile unter Berücksichtigung der Hauptbestandteile, während eine Anzahl nebensächlicher Elemente und die aus Quarz bestehende Gangart nicht im einzelnen aufgeführt wurde.

\begin{tabular}{|c|c|c|}
\hline & $\begin{array}{l}\text { Ursprüngliches } \\
\text { Mineral }\end{array}$ & $\begin{array}{c}\text { Mit Thoulet'scher } \\
\text { Losung behandeltes } \\
\text { Mineral }\end{array}$ \\
\hline $\mathrm{V}_{2} \mathrm{O}_{5}$ & 18,03 & 18,31 \\
\hline$U O_{3}$ & 53,71 & 55,37 \\
\hline $\mathrm{CaO}$. & 5,20 & 1,72 \\
\hline $\mathrm{K}_{2} \mathrm{O}$. & 0,24 & 6,08 \\
\hline $\mathrm{CuO}$. . . . . & 4,16 & 4,22 \\
\hline $\left.\begin{array}{l}\mathrm{SiO}_{2}, \mathrm{P}_{2} \mathrm{O}_{5}, \mathrm{BaO} \\
\mathrm{Al}_{2} \mathrm{O}_{3}, \mathrm{MgO}, \mathrm{Na}_{2} \mathrm{O}\end{array}\right\}$ & 2,45 & 1,91 \\
\hline \multirow{2}{*}{$\mathrm{H}_{2} \mathrm{O}$ und Verlust } & 16,21 & 12,39 \\
\hline & 100,00 & 100,00 \\
\hline
\end{tabular}

Es sei bemerkt, daß das analysierte Material im vorliegenden Falle ein Gemenge war, und daß das Kupfer zum größten Teile, wenn nicht gar vollstāndig einem grünlichen Vanadat angehört, zu welchem wahrscheinlich auch ein Teil des Calciums zu rechnen ist.

Der einzige wesentliche Wechsel in der Zusammensetzung des ursprünglichen Minerals scheint hinsichtlich des Calciums und des Wassers eingetreten zu sein.

Das Molekularverhältnis der Summe $\mathrm{CaO}+\mathrm{K}_{2} \mathrm{O}$ beträgt in beiden Analysen $954: 952$, was auf eine genau äquivalente Ersetzung des Calciums durch Kalium hinweist. Die Abnahme im Wassergehalt bei der mit Thoulet'scher Lösung behandelten Probe erklärt praktisch die hier etwas höheren Werte für Vanadin und Uran. Der Unterschied in den Wasserwerten braucht jedoch nicht wirklich vorhanden zu sein, da man heute weiß, daß der Wassergehalt eines Minerals um einige Prozent schwanken kann, je nachdem, ob die Witterung trocken oder feucht ist. Auffallend ist die Tatsache, daß der Kupfergehalt durch die schwere Lösung anscheinend nicht beeinflußt worden ist. $\mathrm{Ob}$ durch eine längere Behandlung eine vollständige Ver- 
Über eine b. d. Trennung v. Mineralien mittelst schw. Lösgn. zu beacht. Gefahr. 3

drängung des Calciums durch Kalium würde stattgefunden haben, konnte wegen Mangel an Material und Gelegenheit nicht weiter festgestellt werden. Bei früheren Proben des vorbehandelten Materials wurde bemerkt, daß das Calcium nicht völlig ersetzt worden war. Dies stimmt mit der Beobachtung überein, daß das Kupfervanadat jedenfalls Calcium enthält; und da der Kupfergehalt dieses Minerals keine Veränderung erlitten hat, ist es nicht unwahrscheinlich, daß auch die anderen Basen zurückgehalten wurden, welcher Natur sie auch immer seien.

Nach H. E. Merwin zeigte das gelbe Calciummineral nach der Behandlung mit Thoulet'scher Lösung eine Abnahme des Winkels der optischen Axen um 250, verglichen mit dem nicht behandelten Material von annähernd demselben Wassergehalt.

Die im Vorstehenden beschriebene Beobachtung zeigt, wie wichtig es ist, sich beim Gebrauch von schweren Lösungen davon zu überzeugen, daß chemische Veränderungen der oben geschilderten Art nicht vor sich gegangen sind. Es erheben sich nun naturgemäß Zweifel bezüglich der Gültigkeit der für das eine oder andere Mineral bestimmten Zusammensetzung, das vor der Analyse von anderen mittelst schwerer Lösungen getrennt worden war.

Der bei vorstehender Untersuchung verwendete Calcium-Carnotit ist wahrscheinlich mit dem Tuyamayunit von Nenadkewitsch 1) identisch und bildet den Gegenstand einer späteren eingehenden Beschreibung von Seiten des Herrn Merwin und mir.

Bureau of Standards, Washington, D. C., Februar 1913.

1) Bulletin de l'Acad. des Sciences St. Pétersbourg, 1912, 940. 